\title{
Assessment of clinical significance of positive blood cultures of relatively low-virulence isolates
}

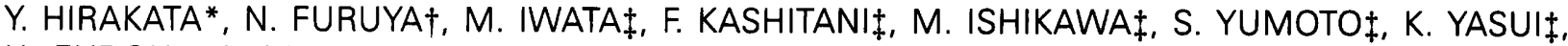 \\ H. ENDOH $\ddagger, A$. MARUI $\ddagger, M$. KAKU§, K. TATEDA $\dagger$ and K. YAMAGUCHI $\ddagger$
}

†Department of Microbiology and $\ddagger$ Clinical Laboratory, Omori Hospital, Toho University School of Medicine, Tokyo 143 and §Department of Laboratory Medicine, Nagasaki University School of Medicine, Nagasaki 852, Japan

\begin{abstract}
In Omori Hospital, Toho University School of Medicine, relatively low-virulence blood isolates, including coagulase-negative staphylococci (CNS), enterococci and nonfermentative gram-negative rods other than Pseudomonas aeruginosa comprised $c$. $60 \%$ of total blood isolates. A retrospective study was conducted to assess their clinical significance by reviewing a total of 91 hospital charts. The physicians' assessments of these positive blood cultures as recorded in the charts were classified into four categories-sepsis, possible sepsis, contamination and no comment. The episodes classified as sepsis accounted for $5.0-19.6 \%$. These episodes were also evaluated by a graded clinical significance score based on multiple factors, including number of positive cultures and clinical signs. The scores for the 91 episodes covered a wide range from 1 to 9 , indicating that both contaminants and causative organisms may have been involved. The episodes judged as sepsis or possible sepsis tended to have higher scores. The scores for the episodes associated with enterococci were also higher than those involving CNS or non-fermentative gram-negative rods. The scores for episodes associated with intravenous hyperalimentation catheters were higher than those not associated with the catheters.
\end{abstract}

\section{Introduction}

Blood culture is a simple procedure and is the best criterion for defining bacteraemia [1]. The isolation of any organism from a blood culture requires careful evaluation by the clinician [2]; however, it is frequently difficult to determine whether blood isolates are the true cause of septicaemia or merely contaminants. When micro-organisms of low virulence, especially skin flora, are isolated from blood cultures, they have been dismissed traditionally as contaminants $[1,3]$. Recently, however, gram-positive cocci have reemerged as the most prevalent cause of hospitalacquired bloodstream infections [4-6]. The trend is related to the increased incidence of bacteraemia due to coagulase-negative staphylococci (CNS) [6-8] and enterococci $[9,10]$. The assessment of positive blood culture seems to have become more difficult.

Received 7 March 1995; revised version accepted 4 Aug. 1995.

*Present address and address for correspondence: Department of Laboratory Medicine, Nagasaki University School of Medicine, Nagasaki 852, Japan.
Positive blood cultures from Omori Hospital, Toho University School of Medicine were examined to assess the clinical significance of relatively lowvirulence blood isolates including CNS. The isolates were evaluated by reviewing hospital charts and by an original graded clinical significance score.

\section{Materials and methods}

\section{Laboratory methods}

Omori Hospital, Toho University School of Medicine, is a 1080-bed hospital. Approximately 2000 blood cultures were examined per year in the clinical laboratory. A one-bottle system with sodium amylosulphate triple-extracted peptone broth (Culture bottle No. 5 Eiken; Eiken Chemical Co., Japan) was routinely used for blood culture. Each bottle, containing $45 \mathrm{ml}$ of broth, was inoculated with $5 \mathrm{ml}$ of blood. Cultures were evaluated daily for 1 week before being deemed negative. Blind subculture was performed every second day. Bacterial isolates were identified by Vitek GramPositive or Gram-Negative Identification cards (Vitek Systems, USA). 


\section{Virulent and relatively low-virulence blood isolates}

Virulent isolates were defined as strains of Staphylococcus aureus, Streptococcus pneumoniae, Str. pyogenes, Haemophilus spp., members of Enterobacteriaceae, Pseudomonas aeruginosa, Bacteroides spp. and other anaerobes, or Candida albicans. The other isolates, including CNS, enterococci and nonfermentative gram-negative rods other than $P$. aeruginosa, were defined as relatively low-virulence isolates.

\section{Review of hospital charts}

The hospital charts of patients reported to have positive blood cultures between January and December 1988 were reviewed along with those from the same period of 1992. A septic episode of bacteraemia was defined as the first positive blood culture or a new positive blood culture occurring $48 \mathrm{~h}$ after the preceding positive culture [11]. Cases of polymicrobic bacteraemia or those with insufficient information in the charts were excluded from analysis. Cases of fungaemia were also excluded from this study. The information obtained included the patient's name, identification number, age, sex, date of admission, date of positive blood culture, primary diagnosis, suspected risk factors, diagnosis or comments on positive blood culture by the physician, time course of body temperature, blood pressure, urine output, laboratory data including peripheral leucocyte count, platelets, C-reactive protein (CRP), erythrocyte sedimentation rate (ESR), levels of fibrinogen/fibrin degradation products and prognosis.

\section{Clinical significance score}

The clinical significance of the blood isolates was determined with a graded score. A basal score was determined by the number of positive and total culture bottles as shown in Table 1. Additional scores were given if the septic episode fulfilled each of the following criteria: (i) the same organism grown from another site, as determined by the species and the results of antibiotic susceptibility; (ii) a clinical judgment of sepsis or possible sepsis by a physician (score was not given for episodes without comments by physicians); (iii) presence of iatrogenic risk factors such as intravenous catheter, or administration of immunosuppressive agents; (iv) inflammatory response (at least two of the following: temperature $>38^{\circ} \mathrm{C}$, increase in leucocytes, increase in CRP, accelerated ESR); (v) deteriorating general status (at least one of the following: low blood pressure, decrease in urine

Table 1. Basal score in a graded score of clinical significance for positive blood culture episodes

\begin{tabular}{lc}
\hline $\begin{array}{l}\text { Number of blood cultures } \\
\text { (positive/total) }\end{array}$ & Basal score \\
\hline $4 / 4,3 / 3,2 / 2$ & 3 \\
$1 / 2,2 / 3,2 / 4$ & 2 \\
$1 / 3$ & 1 \\
\hline
\end{tabular}

output, increase in fibrinogen/fibrin degradation products, decrease in platelets).

\section{Results}

\section{Blood isolates in 1988 and 1992}

The numbers of blood culture samples examined in 1988 and 1992 were 1919 and 1678 per year, respectively. In 1988, a total of 182 isolates was obtained from 139 septic episodes (the rate of positive blood culture was 9.5\%). In 1992, 199 isolates were obtained from 168 septic episodes (12.2\%). The numbers of organisms isolated are summarised in Tables 2 and 3. Low-virulence isolates comprised $64.3 \%$ of total isolates in 1988 and $58.3 \%$ of total isolates in 1992 (Table 3).

\section{Review of positive blood culture episodes}

Distribution of the relatively low-virulence isolates in the septic episodes reviewed is summarised in Table 4. For 1988 and 1992, 51 and 40 septic episodes were reviewed, respectively. CNS, non-fermentative gramnegative rods and enterococci comprised 52.7, 18.7 and $12.1 \%$, respectively, of the positive blood cultures. Table 4 also shows the distribution of the episodes with intravenous hyperalimentation (IVH) catheters.

Table 2. 'Virulent' blood isolates in 1988 and 1992 at Omori Hospital, Toho University School of Medicine

\begin{tabular}{|c|c|c|}
\hline \multirow[b]{2}{*}{ Species } & \multicolumn{2}{|c|}{ Number $(\%)$ of isolates in } \\
\hline & 1988 & 1992 \\
\hline S. aureus & $25(13.7)$ & $47(23.6)$ \\
\hline Str. pneumoniae & $2(1.1)$ & $1(0.5)$ \\
\hline Str. pyogenes & $1(0.6)$ & $0 \quad(0.0)$ \\
\hline Escherichia coli & $10 \quad(5.5)$ & $1 \quad(0.5)$ \\
\hline Klebsiella pneumoniae & $0 \quad(0.0)$ & $4 \quad(2.0)$ \\
\hline Enterobacter spp. & $7 \quad(3.9)$ & $8 \quad(4.0)$ \\
\hline Citrobacter spp. & $1(0.6)$ & $0 \quad(0.0)$ \\
\hline Serratia spp. & $5 \quad(3.9)$ & $(1.0)$ \\
\hline Other Enterobacteriaceae & $0 \quad(0.0)$ & $2(1.0)$ \\
\hline Haemophilus spp. & $2(1.1)$ & $6 \quad(3.0)$ \\
\hline$P$. aeruginosa & $4 \quad(2.2)$ & $(3.5)$ \\
\hline Other anaerobes & $3(1.7)$ & $(0.5)$ \\
\hline Bacteroides spp. & $4 \quad(2.2)$ & $1 \quad(0.5)$ \\
\hline Candida albicans & $1 \quad(0.6)$ & $3(1.51)$ \\
\hline Total & $65(35.7)$ & $83(41.7)$ \\
\hline
\end{tabular}

Table 3. Relatively low-virulence blood isolates in 1988 and 1992 at Omori Hospital, Toho University School of Medicine

\begin{tabular}{|c|c|c|}
\hline \multirow[b]{2}{*}{ Micro-organism } & \multicolumn{2}{|c|}{ Number $(\%)$ of isolates } \\
\hline & 1988 & 1992 \\
\hline Coagulase-negative staphylococci & $55(30.2)$ & $60(30.2)$ \\
\hline Enterococci & $19(10.4)$ & $12(6.0)$ \\
\hline Other gram-positive cocci & $5(2.8)$ & $11 \quad(5.5)$ \\
\hline Gram-positive rods & $12(6.6)$ & $4(2.0)$ \\
\hline Non-fermentative gram-negative rods* & $13(7.1)$ & $16(8.0)$ \\
\hline Propionibacterium spp. & $7 \quad(3.9)$ & (4.0) \\
\hline Fungi other than $C$. albicans & $6 \quad(3.3)$ & $5 \quad(2.5)$ \\
\hline Total & $117(64.3)$ & $116(58.3)$ \\
\hline
\end{tabular}

*Other than $P$. aeruginosa. 
Table 4. Distribution of isolates from review of blood culture-positive episodes

\begin{tabular}{|c|c|c|c|}
\hline \multirow[b]{2}{*}{ Micro-organism } & \multicolumn{3}{|c|}{$\begin{array}{c}\text { Number of episodes } \\
\text { (number involving IVH catheters) }\end{array}$} \\
\hline & 1988 & 1992 & Total \\
\hline Coagulase-negative staphylococci & $24 \quad(7)$ & $24 \quad(8)$ & $48(15)$ \\
\hline Enterococci & 8 (3) & 3 (2) & $11(5)$ \\
\hline Other gram-positive cocci & $2(0)$ & $0(0)$ & $2(0)$ \\
\hline Gram-positive rods & $6(2)$ & 1 (1) & 7 (3) \\
\hline Non-fermentative gram-negative rods* & 8 (5) & $9(2)$ & 17 (7) \\
\hline Propionibacterium spp. & 3 (1) & $3(2)$ & $6(3)$ \\
\hline Total & $51(18)$ & $40(15)$ & $91(33)$ \\
\hline
\end{tabular}

*Other than $P$. aeruginosa, including six strains of $P$. cepacia, one strain of Xanthomonas maltophilia and one strain of Acinetobacter calcoaceticus in 1988, and eight strains of $P$. cepacia and one strain of Alcaligenes faecalis in 1992.

\section{Classification of assessment of positive blood cultures by physicians}

Comments by the physicians were classified into four categories-sepsis, possible sepsis, contamination and no comment. The episodes with 'no comment' included patients who died before a blood culture report was made. The episodes classified as sepsis comprised $19.6 \%$ in 1988 , but this significantly decreased to $5.0 \%$ in 1992 ( $p<0.05$ by $\chi^{2}$ test). In contrast, the episodes judged as contamination or listed as no comment increased from $58.0 \%$ in 1988 to $70.1 \%$ in 1992 (not statistically significant). Overall, no comment was noted in $c .50 \%$ of the septic episodes.

\section{Clinical significance score}

Fig. 1 shows the distribution of clinical significance scores in the episodes with relatively low-virulence

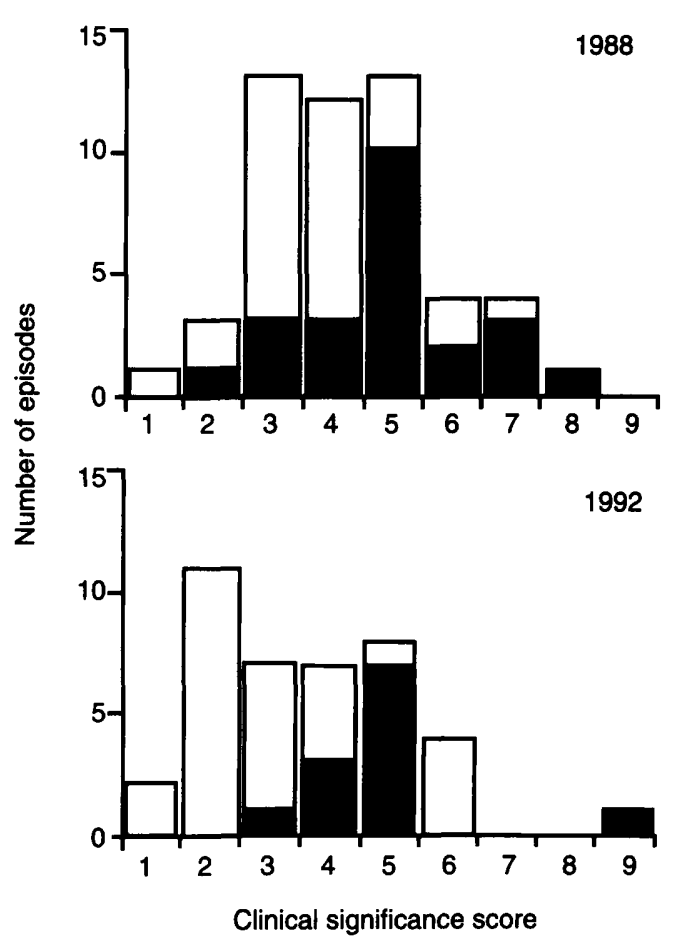

Fig. 1. Distribution of clinical significance scores in positive blood cultures due to relatively low-virulence isolates: $\square$, episodes classified as sepsis or possible sepsis; $\square$, episodes classified as contamination or listed as no comment. isolates. The scores were distributed over a wide range, from 1 to 9. In 1992, the distribution of scores shifted to a lower range. The septic episodes associated with enterococci seemed to have higher scores when compared with those associated with CNS and nonfermentative gram-negative rods. However, the scores for episodes due to CNS, enterococci and nonfermentative gram-negative rods other than $P$. aeruginosa fell over a wide range (Fig. 2). In contrast, the scores for episodes due to virulent isolates including S. aureus (three episodes), Str. pneumoniae (one), Klebsiella pneumoniae (two), Enterobacter aerogenes (one) and P. aeruginosa (one) were distributed from 7 to 9. Fig. 3 shows that the scores of patients with IVH catheters in the episodes with relatively low-virulence isolates were apparently higher than those of patients without IVH catheters.

\section{Discussion}

This study showed that the assessment of positive blood cultures may be very difficult in $60 \%$ of positive blood cultures, as the isolates can cause true bacter-

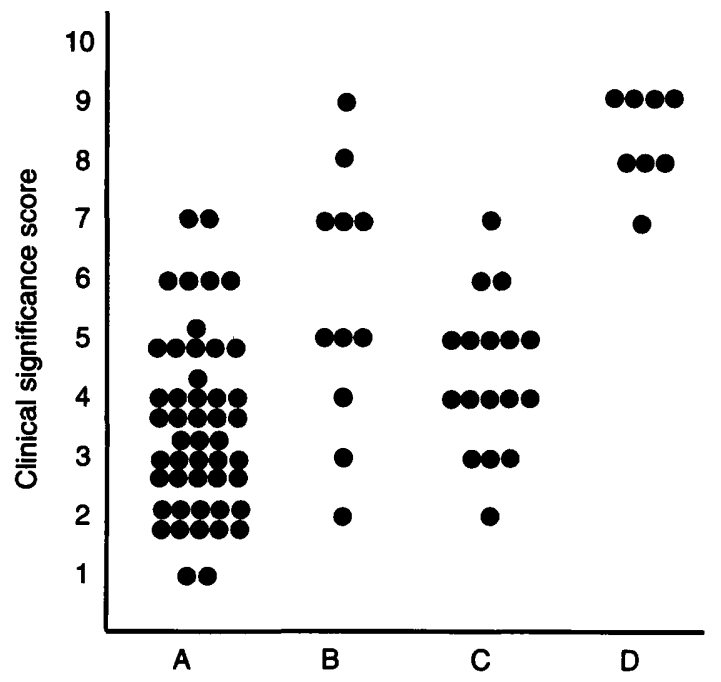

Fig. 2. Comparison of distribution of clinical significance scores in positive blood cultures due to (A) coagulasenegative staphylococci, (B) enterococci, (C) non-fermentative gram- negative rods (except for $P$. aeruginosa) and (D) virulent isolates. 


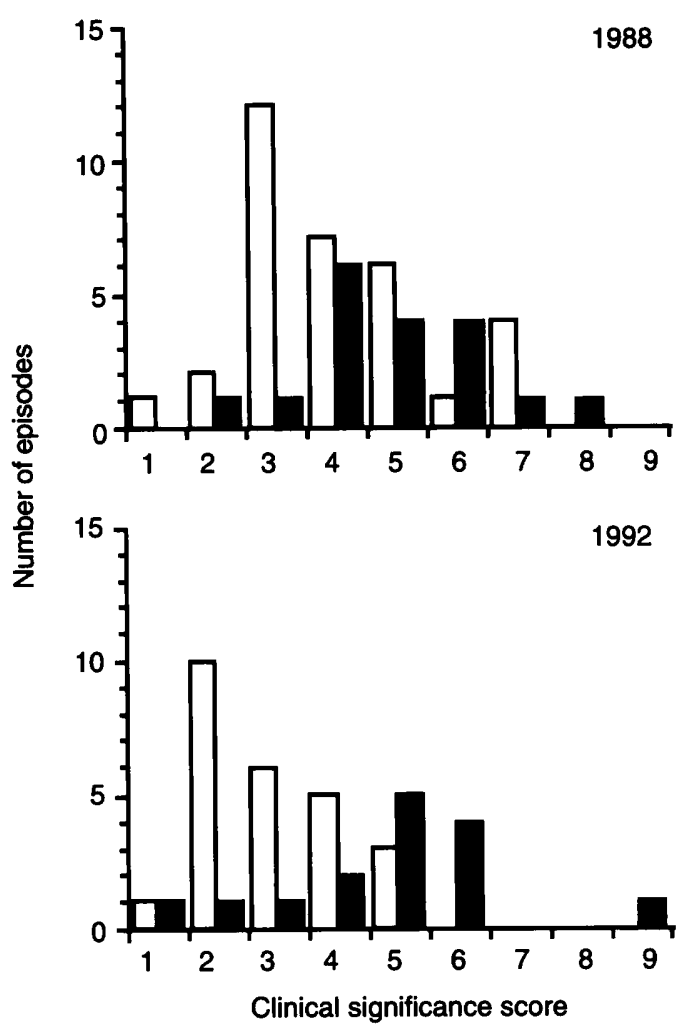

Fig. 3. Comparison of distribution of clinical significance scores between patients with $(\square)$ and without $(\square)$ an IVH catheter in the episodes with relatively lowvirulence isolates.

aemia as well as simple contamination. There are several published reports in which positive blood cultures were evaluated to determine whether they represented true infection or contamination [3,11-13]. The present study evaluated the clinical significance of positive blood cultures with an original grading system. All blood culture-positive episodes with pathogenic isolates showed high scores. These organisms may cause severe inflammatory response in hosts and often are isolated repeatedly as they are usually not contaminants. In consequence they receive high scores. In contrast, scores in the episodes with relatively lowvirulence isolates were distributed over a wide range, from 1 to 9 , indicating that both contaminants and causative organisms may be involved.

The clinical significance of low-virulence isolates was apparently higher in patients with an IVH catheter than in those without (Fig. 3). The IVH catheter itself facilitates bacterial invasion into the bloodstream and often develops a core of biofilm [14]. Many recent studies have shown that CNS are the leading cause of infections related to intravascular devices [15-18]. In the review of the clinical charts, no comments by the physician requesting blood isolates were found in over half of the cases. We speculate that delay in reporting laboratory results may contribute to this. Although physicians have to commence therapy as soon as possible in septic patients, it usually takes $>2$ days to make final reports of blood cultures. The results were reported after the death of the patients in seven of 91 episodes reviewed.

The number of blood cultures/year in our hospital is very low. It is possible that the low positive rate of blood culture and the length of time for the results to become available are reasons for the small numbers of blood cultures taken in our hospital. Moreover, as physicians frequently made no comments on the results, it seems necessary to improve the blood culture system as well as to ensure that the significance of blood cultures is well understood by both clinicians and technicians.

\section{References}

1. Yagupsky P, Nolte FS. Quantitative aspects of septicemia. Clin Microbiol Rev 1990; 3: 269-279.

2. Robert FJ. A review of positive blood cultures: identification and source of microorganisms and patterns of sensitivity to antibiotics. Rev Infect Dis 1980; 2: 329-339.

3. MacGregor RR, Beaty HN. Evaluation of positive blood cultures. Guidelines for early differentiation of contaminated from valid positive cultures. Arch Intern Med 1972; 130: 84-87.

4. Donowitz LG, Haley CE, Gregory WW, Wenzel RP. Neonatal intensive care unit bacteremia: emergence of gram-positive bacteria as major pathogens. Am J Infect Control 1987; 15 $141-147$.

5. Landry SL, Kaiser DL, Wenzel RP. Hospital stay and mortality attributed to nosocomial enterococcal bacteremia: a controlled study. Am J Infect Control 1989; 17: 323-329.

6. Martin MA, Pfaller MA, Wenzel RPV. Coagulase-negative staphylococcal bacteremia - mortality and hospital stay. Ann Intern Med 1989; 110: 9-16.

7. Pfaller MA, Herwaldt LA. Laboratory, clinical, and epidemiological aspects of coagulase-negative staphylococci. Clin Microbiol Rev 1988; 1: 281-299.

8. Stillman RI, Wenzel RP, Donowitz LG. Emergence of coagulase-negative staphylococci as major nosocomial bloodstream pathogens. Infect Control 1987; 8: 108-112.

9. Barrall DT, Kenney PR, Slotman GJ, Burchard KW. Enterococcal bacteremia in surgical patients. Arch Surg 1985; 120: $57-63$.

10. Gullberg RM, Homann SR, Phair JP. Enterococcal bacteremia: analysis of 75 episodes. Rev Infect Dis 1989; 11: 74-85.

11. Weinstein MP, Reller LB, Murphy JR, Lichtenstein KA. The clinical significance of positive blood cultures: a comprehensive analysis of 500 episodes of bacteremia and fungemia in adults. I. Laboratory and epidemiologic observations. Rev Infect Dis 1983; 5: 35-53.

12. Roberts FJ, Geere IW, Coldman A. A three-year study of positive blood cultures, with emphasis on prognosis. Rev Infect Dis 1991; 13: 34-46.

13. Ishak MA, Gröschel DHM, Mandell GL, Wenzel RP. Association of slime with pathogenicity of coagulase-negative staphylococci causing nosocomial septicemia. J Clin Microbiol 1985; 22: 1025-1029.

14. Costerton JW, Cheng KJ, Geesey GG et al. Bacterial biofilms in nature and disease. Annu Rev Microbiol 1987; 41: 435-464.

15. Moyer MA, Edwards LD, Farley L. Comparative culture methods on 101 intravenous catheters. Routine, semiquantitative, and blood cultures. Arch Intern Med 1983; 143: 66-69.

16. Press OW, Ramsey PG, Larson EB, Fefer A, Hickman RO Hickman catheter infections in patients with malignancies. Medicine (Baltimore) 1984; 63: 189-200.

17. Sitges-Serra A, Puig P, Jaurrieta E, Garau J, Alastrue A, Sitges-Creus A. Catheter sepsis due to Staphylococcus epidermidis during parenteral nutrition. Surg Gynecol Obstet 1980; 151: 481-483.

18. Wilson WR, Danielson GK, Giuliani ER, Geraci JE. Prosthetic valve endocarditis. Mayo Clin Proc 1982; 57: 155-161. 